\title{
Gravity-scalar fluctuations in holographic RG flow geometries
}

\author{
G. Arutyunov ${ }^{\mathrm{a}, 1}, \mathrm{~S}$. Frolov ${ }^{\mathrm{b}, 1}, \mathrm{~S}$. Theisen ${ }^{\mathrm{a}}$ \\ ${ }^{a}$ Sektion Physik, Universität München, Theresienstr. 37, D-80333 München, Germany \\ ${ }^{\mathrm{b}}$ Department of Physics and Astronomy, University of Alabama, Box 870324, Tuscaloosa, AL 35487-0324, USA
}

Received 2 May 2000; accepted 6 June 2000

Editor: P.V. Landshoff

\begin{abstract}
We study five-dimensional gravity models with non-vanishing background scalar fields which are dual to non-conformal boundary field theories. We develop a procedure to decouple the graviton fluctuations from the scalar ones and apply it to the simplest case of one scalar field. The quadratic action for the decoupled scalar fluctuations has a very simple form and can be used to compute two-point functions. We perform this computation for the two examples of background RG flow recently considered by DeWolfe and Freedman and find physically reasonable results. (c) 2000 Elsevier Science B.V. All rights reserved.
\end{abstract}

\section{Introduction}

The AdS/CFT correspondence [1-3] provides a powerful method for computing correlation functions of gauge invariant operators in $\mathscr{N}=4$ supersymmetric Yang-Mills theory (YM) in four dimensions at large $N$ and at strong 't Hooft coupling. A question of obvious interest is to extend the correspondence and in particular the prescription for computing Green functions to a general class of theories without (or with spontaneously broken) superconformal invariance. Such theories have a renormalization group flow and they generically arise either from deforming YM away from the conformal point by adding to

E-mail addresses: arut@theorie.physik.uni-muenchen.de (G. Arutyunov), frolov@bama.ua.edu (S. Frolov), theisen@theorie.physik.uni-muenchen.de (S. Theisen).

${ }^{1}$ On leave of absence from Steklov Mathematical Institute, Gubkin str. 8, GSP-1, 117966, Moscow, Russia. the Lagrangian IR relevant perturbations [4-15] or by giving vacuum expectation values to scalar fields [16-20]. In the gauged supergravity description the corresponding bulk geometries are supported in both cases by 4-dimensional Poincaré invariant kink solutions approaching asymptotically $\mathrm{AdS}$ space (see [21] for a recent extensive review of the subject).

Recently a gravity computation of some two-point correlation functions in field theories with renormalization group flow was presented in [22]. This computation amounts to the study of the coupled gravity-scalar equations describing fluctuations of the scalars and the metric around a kink solution. The background scalars naturally fall into two classes: the scalars with a non-trivial dependence on the fifth dimension which, in the terminology of [22], are called "active", and the constant (or vanishing) "inert' scalars. In [22] striking differences between the expected correlation functions of YM operators dual to inert and to active scalars were observed. The 
correlation functions of the operators dual to the inert scalars are straightforward to compute and they provide a consistent description of the spectrum in the boundary field theory. Quite opposite, the correlation functions of the operators dual to active scalars were then found to be physically unreasonable. The authors of [22] suggested that this might be due to the presence of the metric singularity in the interior, which in turn might invalidate the standard AdS prescription for computing correlation functions. Adding the standard supplementary gravity boundary terms did not clarify the situation.

In this note we reconsider the two-point correlation functions for operators dual to active scalars. We use the Hamiltonian formulation of the AdS / CFT correspondence [23] to deal with boundary terms and the standard prescription for computing correlation functions in the gravity approximation $[2,3]$. Another point of deviation from the analysis in [22] is our choice of gauge.

We start by analyzing the quadratic action for fluctuations of the scalar fields and the metric near their background values. All the boundary terms one could add to the gravity action are unambiguously fixed by the Hamiltonian version of the AdS/CFT correspondence [23] that requires the gravity action to be schematically of the form $\int d t(p \dot{q}-H(p, q))$, where $H$ is the Hamiltonian and $t$, the coordinate in the bulk direction, plays the role of the time. For the quadratic action this prescription implies that it does not contain any gravity or scalar fields with second derivative in the bulk direction. All such terms are integrated by parts and arising boundary terms are simply discarded.

For the sake of simplicity we consider the case of a unique active scalar $\bar{\phi}$ and pick up the "almost" radial gauge for which the scalar fluctuation $\phi$ is set to zero. The residual gauge symmetry is enough to decouple the transverse components of the graviton and it leads to the conservation law for the stress-energy tensor in the boundary field theory. Except for the traceless transverse graviton the only physical degree of freedom is then the trace of the graviton $h$ for which we obtain a very simple action, basically due to the existence of a superpotential.

The trace of the graviton is usually viewed as the gravity field dual to the trace of the stress-energy tensor in the field theory away from the conformal point. However, we show that by using a field redefinition one can recast the action for $h$ into the standard action for a scalar field $s$ in the kink background with some complicated potential. Depending of the form of the kink solution, the scalar $s$ may then be naturally interpreted as the dual either to the YM operator invoking RG flow or to the corresponding field theory operator with a non-trivial vacuum expectation value. The relation between $h$ and $s$ appears to be $h \sim \beta^{-1} s$, where $\beta$ is the holographic beta function introduced in [10]. We believe that a similar relation also occurs for general backgrounds with many active scalars [24].

We then consider the same two kink solutions as in [22]. The first one corresponds to $\mathscr{N}=4 \mathrm{SYM}$, perturbed by an operator of dimension 3, that flows in the IR to $\mathscr{N}=1 \mathrm{SYM}$. The second kink involves one active scalar from the $\mathbf{2 0}$ of $S O(6)$ and the corresponding dual flow describes the states on the Coulomb branch of $\mathscr{N}=4$ SYM, which is parametrized by the vacuum expectation value of one of the operators $O_{2}=\operatorname{tr}\left(X^{(I} X^{J)}\right)$, where $X^{I}$ are YM scalars.

Evaluating the on-shell action for these supergravity solutions we find that in both cases the two-point functions exhibit the same behaviour as was found for the inert scalars in $[18,22]$. Indeed, in the first case we get a discrete spectrum while in the second example the spectrum is continuous with a mass gap.

\section{Gravity / active scalar system}

Consider $(d+1)$-dimensional gravity, described by a metric $G_{\mu \nu}$ with signature $(1,1, \ldots, 1,-1)$, minimally coupled to scalar fields $\varphi^{I}$. The action is of the form ${ }^{2}$

$S=\int d^{d+1} x \sqrt{-G}\left(\frac{1}{4} R-\frac{1}{2} G^{\mu \nu} \partial_{\mu} \varphi \partial_{\nu} \varphi^{I}-V(\varphi)\right)$,

\footnotetext{
${ }^{2}$ Our conventions are: $\left[\nabla_{\mu}, \nabla_{\nu}\right] V_{\rho}=R_{\mu \nu \rho}{ }^{\sigma} V_{\sigma}$ and $R_{\mu \rho}=R_{\mu \nu \rho}{ }^{\nu}$.
} 
$V(\varphi)$ is the scalar potential. The equations of motion that follow from this action are

$$
\begin{aligned}
& R_{\mu \nu}-\frac{1}{2} G_{\mu \nu} R-2 \partial_{\mu} \varphi \partial_{\nu} \varphi^{I}+G_{\mu \nu} \partial_{\rho} \varphi^{I} \partial^{\rho} \varphi^{I} \\
& \quad+2 G_{\mu \nu} V=0, \\
& \nabla^{\mu} \partial_{\mu} \varphi^{I}-\frac{\partial V}{\partial \varphi^{I}}=0 .
\end{aligned}
$$

They imply

$$
\begin{aligned}
& R-2 \partial_{\rho} \varphi^{\partial}{ }^{\rho} \varphi^{I}=4 \frac{d+1}{d-1} V, \\
& R_{\mu \nu}-2 \partial_{\mu} \varphi^{J} \partial_{\nu} \varphi^{I}=\frac{4 V}{d-1} G_{\mu \nu} .
\end{aligned}
$$

Let $g_{\mu \nu}$ and $\bar{\phi}^{I}$ be solutions of the equations of motion and decompose $G_{\mu \nu}$ and $\varphi$ around their background values

$G_{\mu \nu}=g_{\mu \nu}+h_{\mu \nu}, \quad \varphi^{I}=\bar{\phi}^{I}+\phi^{I}$.

Discarding total derivative terms, we find the following quadratic action for the fluctuations

$$
\begin{aligned}
S_{2}= & \int d^{d+1} x \sqrt{-g}\left[\frac { 1 } { 4 } \left(-\frac{1}{4} \nabla_{\rho} h_{\mu \nu} \nabla^{\rho} h^{\mu \nu}\right.\right. \\
& +\frac{1}{2} \nabla_{\rho} h_{\mu \nu} \nabla^{\mu} h^{\rho \nu}-\frac{1}{2} \nabla^{\nu} h \nabla^{\mu} h_{\mu \nu} \\
& \left.+\frac{1}{4} \nabla_{\mu} h \nabla^{\mu} h+\frac{2 V}{d-1} h_{\mu \nu}^{2}-\frac{V}{d-1} h^{2}\right) \\
& +h^{\mu \nu} \nabla_{\mu} \phi^{I} \nabla_{\nu} \bar{\phi}^{I}+\frac{1}{2} \nabla_{\mu} h \phi^{I} \nabla^{\mu} \bar{\phi}^{I} \\
& \left.-\frac{1}{2} \nabla_{\mu} \phi^{I} \nabla^{\mu} \phi^{I}-\frac{1}{2} V_{I J} \phi^{I} \phi^{J}\right] .
\end{aligned}
$$

The covariant derivative $\nabla_{\mu}$ is with respect to the background metric $g_{\mu \nu}$ which is also used to raise and lower indices, $h=h_{\mu}^{\mu}$, and we have introduced the notation

$V_{I}=\left.\frac{\partial V}{\partial \varphi^{I}}\right|_{\varphi=\bar{\phi}}, \quad V_{I J}=\left.\frac{\partial^{2} V}{\partial \varphi \partial \varphi^{J}}\right|_{\varphi=\bar{\phi}}$.

Within the context of the AdS /CFT correspondence, we are interested in background solutions which respect $d$-dimensional Poincaré invariance. Thus, we make an ansatz for the background solving (2.2) of the form

$d s^{2}=d x_{0}^{2}+e^{2 A\left(x_{0}\right)} \eta_{i j} d x^{i} d x^{j}$,

where $\eta_{i j}$ is the Minkowski metric and take $\bar{\phi}$ to depend only on $x_{0}$.

The analysis of the action and the equations of motion is complicated by the fact that the scalar fields couple to the graviton already in the quadratic action. Therefore, the spectrum of the theory on this background cannot be readily read off the action or the equations. However, the problem of finding the spectrum of the theory can be simplified by an appropriate gauge choice. The quadratic action and the equations of motion are invariant under gauge transformations induced by reparametrizations:

$\delta h_{\mu \nu}=\nabla_{\mu} \zeta_{\nu}+\nabla_{\nu} \zeta_{\mu}, \quad \delta \phi^{I}=\zeta^{\mu} \nabla_{\mu} \bar{\phi}^{I}$.

One usually imposes the temporal gauge $h_{0 \mu}=0$ and solves the constraints, i.e. the equations of motion for $h_{0 \mu}$. This was done in [22]. However, this gauge choice leads to a very complicated system of equations and, moreover, it was only possible to derive a third-order equation for scalar fields. On the other hand, the gauge transformations (2.6) of scalar fields show that on backgrounds with at least one nonvanishing field $\bar{\phi}^{1} \equiv \bar{\phi}$ one can impose the almost temporal gauge $h_{0 i}=0=\phi$. This gauge choice is very natural, because the combination $\tilde{h}_{00} \equiv h_{00}-$ $2 \nabla_{0}\left(\phi / \nabla_{0} \bar{\phi}\right)$ is gauge invariant, and one could express the action and the equations of motion in terms of $\tilde{h}_{00}$ rather than $h_{00}$. Then only the scalar fields change under gauge transformations.

For the sake of simplicity we restrict ourselves to considering the simplest case of one active scalar. Then the action depends only on $h_{i j}$ and $h_{00}$. It is convenient to introduce $t_{i j}$ via

$h_{i j}=e^{2 A} t_{i j}, \quad h^{i j}=e^{-2 A} t^{i j}, \quad t \equiv t_{i}^{i}=t_{i j} \eta^{i j}$.

In what follows we will not distinguish upper and lower indices. It is straightforward to derive

$$
\begin{aligned}
& \nabla_{0} h_{00}=\partial_{0} h_{00}, \quad \nabla_{i} h_{00}=\partial_{i} h_{00}, \quad \nabla_{0} h_{0 i}=0, \\
& \nabla_{i} h_{0 j}=e^{2 A \partial_{0} A\left(\eta_{i j} h_{00}-t_{i j}\right),} \\
& \nabla_{0} h_{i j}=e^{2 A} \partial_{0} t_{i j}, \quad \nabla_{i} h_{k l}=e^{2 A_{i}} t_{k l}
\end{aligned}
$$


and to cast the action (2.4) in the form

$$
\begin{aligned}
S_{2}= & \int d^{d+1} x \frac{1}{4} e^{d A}\left[-\frac{1}{4}\left(\partial_{0} t_{i j}\right)^{2}+\frac{1}{4}\left(\partial_{0} t\right)^{2}\right. \\
& -\frac{1}{4} e^{-2 A}\left(\partial_{i} t_{k l}\right)^{2}+\frac{1}{2} \partial_{0}^{2} A t_{i j}^{2}+\frac{d}{2}\left(\partial_{0} A\right)^{2} t_{i j}^{2} \\
& +\frac{1}{2} e^{-2 A} \partial_{i} t_{k l} \partial_{k} t_{i l}-\frac{1}{4} \partial_{0}^{2} A t^{2}-\frac{d}{4}\left(\partial_{0} A\right)^{2} t^{2} \\
& +\frac{1}{2} e^{-2 A} t \partial_{i} \partial_{j} t_{i j}+\frac{1}{4} e^{-2 A}\left(\partial_{i} t\right)^{2}+\frac{2 V}{d-1} t_{i j}^{2} \\
& -\frac{V}{d-1} t^{2} \\
& +h_{00}\left(\frac{1-d}{2} \partial_{0} A \partial_{0} t-\frac{1}{2} \partial_{0}^{2} A t-\frac{d}{2}\left(\partial_{0} A\right)^{2} t\right. \\
& +\frac{1}{2} e^{-2 A \partial_{i} \partial_{j} t_{i j}}-\frac{1}{2} e^{\left.-2 A_{\partial}^{2} t-\frac{2 V}{d-1} t\right)} \\
& \left.+h_{00}\left(\frac{d}{4} \partial_{0}^{2} A+\frac{d^{2}}{4}\left(\partial_{0} A\right)^{2}+\frac{V}{d-1}\right) h_{00}\right] .
\end{aligned}
$$

We see from this action that $h_{00}$ is a non-dynamical field, and can thus be integrated out. On the other hand we have the constraints that follow from the $h_{0 i}$ equations of motion and from the equation for $\phi$. They are

$$
\begin{aligned}
& (1-d) \partial_{0} A \partial_{i} h_{00}+\partial_{0}\left(\partial_{i} t-\partial_{j} t_{j i}\right)=0, \\
& \partial_{0} h_{00} \partial_{0} \bar{\phi}+2\left(d \partial_{0} A \partial_{0} \bar{\phi}+\partial_{0}^{2} \bar{\phi}\right) h_{00}-\partial_{0} t \partial_{0} \bar{\phi}=0 .
\end{aligned}
$$

These constraints allow us to express $h_{00}$ through $t_{i j}$ and therefore, they should be compatible with the equation of motion for $h_{00}$ that follows from (2.7).

From here on we restrict ourselves to the most interesting case $d=4$, and to a potential $V(\varphi)$ which can be derived from a superpotential $W(\varphi)$,

$V(\phi)=\frac{g^{2}}{8}\left(\frac{\partial W}{\partial \phi}\right)^{2}-\frac{g^{2}}{3} W^{2}$.

All explicitly known backgrounds are obtained from such a potential. One can show [8], that any solution to the equations

$$
\partial_{0} A=-\frac{g}{3} W, \quad \partial_{0} \bar{\phi}=\frac{g}{2} \frac{\partial W}{\partial \bar{\phi}}, \quad g=\frac{2}{L},
$$

also satisfies the equations of motion (2.2). The length scale $L$ is related to the cosmological constant $\Lambda$ via $\Lambda=-12 / L^{2}=4 V(\varphi=0)$. It is not difficult to verify that these relations lead to the identity

$\partial_{0}^{2} A+4\left(\partial_{0} A\right)^{2}+\frac{4}{3} V=0$.

This identity simplifies the action (2.7) considerably and it now takes the form

$$
\begin{aligned}
S_{2}= & \int d^{5} x \frac{1}{4} e^{4 A}\left[-\frac{1}{4}\left(\partial_{0} t_{i j}\right)^{2}+\frac{1}{4}\left(\partial_{0} t\right)^{2}\right. \\
& -\frac{1}{4} e^{-2 A}\left(\partial_{i} t_{k l}\right)^{2}+\frac{1}{2} e^{-2 A} \partial_{k} t_{k l} \partial_{i} t_{i l} \\
& +\frac{1}{2} e^{-2 A} t \partial_{i} \partial_{j} t_{i j}+\frac{1}{4} e^{-2 A}\left(\partial_{i} t\right)^{2} \\
& +h_{00}\left(-\frac{3}{2} \partial_{0} A \partial_{0} t+\frac{1}{2} e^{-2 A} \partial_{i} \partial_{j} t_{i j}\right. \\
& -\frac{1}{2} e^{\left.\left.-2 A \partial_{i}^{2} t\right)-V h_{00}^{2}\right] .}
\end{aligned}
$$

It is well-known (see, e.g. [23]) that the transverse traceless components of the metric fluctuations decouple, and are described by the same equation as a free minimally-coupled massless scalar in the background (2.5). The physical reason for the decoupling is that due to the Lorentz invariance the boundary stress tensor is conserved and, therefore, only the transverse traceless components can couple to it. For this reason it is convenient to introduce the following decomposition of the graviton

$t_{i j}=t_{i j}^{\perp}+t_{i j}^{\|}+\frac{1}{4} h \eta_{i j}-\partial_{i} \partial_{j} H$.

Here $t_{i j}^{\perp}$ is the traceless transverse part and $t_{i j}^{\|}$is a traceless longitudinal part given by

$t_{i j}^{\|}=\frac{\partial_{i}}{\square} \partial_{k} t_{j k}+\frac{\partial_{j}}{\square} \partial_{k} t_{i k}-2 \frac{\partial_{i} \partial_{j}}{\square^{2}} \partial_{k} \partial_{m} t_{k m} ;$

it satisfies $\partial_{i} \partial_{j} t_{i j}^{\|}=0$.

Substituting this decomposition in the action one can easily see that the transverse traceless components do decouple, and in what follows we will drop 
them. Moreover, the longitudinal traceless components also decouple and the only remaining coupled fields are $h$ and $H$.

To analyze their action we introduce their Fourier transforms

$t_{i j}\left(x_{0}, x\right)=\frac{1}{4 \pi^{2}} \int d^{4} p e^{i p x} t_{i j}\left(x_{0}, p\right)$,

$h_{00}\left(x_{0}, x\right)=\frac{1}{4 \pi^{2}} \int d^{4} p e^{i p x} h_{00}\left(x_{0}, p\right)$.

In momentum space the constraints (2.8) take the form

$-3 \partial_{0} A h_{00} p_{i}+\frac{3}{4} p_{i} \partial_{0} h-p_{j} \partial_{0} t_{i j}^{\|}=0$.

From here one finds that

$\partial_{0} A h_{00}=\frac{1}{4} \partial_{0} h$.

and $t_{i j}^{\|}$does not depend on $x_{0}$. Thus, $t_{i j}^{\|}$are not dynamical modes and do not couple to any operators in the boundary theory. They may therefore be omitted. form

We are left with the graviton modes $t_{i j}$ of the

$t_{i j}=\frac{1}{4} \eta_{i j} h+p_{i} p_{j} H$,

whose dynamics is described by the action

$$
\begin{aligned}
S_{2}= & \int d x_{0} d^{4} p \frac{1}{4} e^{4 A}\left[\frac{3}{16}\left(\partial_{0} h\right)^{2}+\frac{3}{8} p^{2} \partial_{0} h \partial_{0} H\right. \\
& +\frac{3}{32} e^{-2 A} p^{2} h^{2} \\
& +h_{00}\left(-\frac{3}{2} \partial_{0} A\left(\partial_{0} h+p^{2} \partial_{0} H\right)+\frac{3}{8} e^{-2 A} p^{2} h\right) \\
& \left.-V h_{00}^{2}\right] .
\end{aligned}
$$

Making the following shift of $h_{00}$

$h_{00} \rightarrow h_{00}+\frac{\partial_{0} h}{4 \partial_{0} A}$, we rewrite (2.16) as

$$
\begin{aligned}
S_{2}= & \int d x_{0} d^{4} p \frac{1}{4} e^{4 A}\left[\frac{3}{16}\left(\partial_{0} h\right)^{2}\right. \\
& -\frac{3}{2} p^{2} \partial_{0} A h_{00} \partial_{0} H+\frac{3}{32} e^{-2 A} p^{2} h^{2} \\
& +\left(h_{00}+\frac{\partial_{0} h}{4 \partial_{0} A}\right) \\
& \times\left(-\frac{3}{2} \partial_{0} A \partial_{0} h+\frac{3}{8} e^{-2 A} p^{2} h\right) \\
& \left.-V\left(h_{00}+\frac{\partial_{0} h}{4 \partial_{0} A}\right)^{2}\right] .
\end{aligned}
$$

Thus $h_{00}$ is a momentum for $H$ and the constraint (2.15), which, after the shift (2.17) reads $h_{00}=0$, shows that $H$ is not a dynamical field. So we can set $h_{00}=0$ and obtain, using again (2.12), the final action for $h$

$$
\begin{aligned}
S_{2}= & \int d x_{0} d^{4} p \frac{1}{4} e^{4 A} \cdot \frac{3}{64} \frac{\partial_{0}^{2} A}{\left(\partial_{0} A\right)^{2}} \\
& \times\left(\left(\partial_{0} h\right)^{2}+e^{-2 A} p^{2} h^{2}\right) .
\end{aligned}
$$

Some comments are in order. The action (2.19) has the correct overall sign as $\partial_{0}^{2} A$ is always negative [8]. The terms in parentheses are exactly the same as for the transverse traceless components. Actually the only difference between the action (2.19) and the action for the transverse traceless components is in the factor

$$
\begin{aligned}
-\frac{3}{16} \frac{\partial_{0}^{2} A}{\left(\partial_{0} A\right)^{2}} & =\frac{9}{32}\left(\frac{\partial}{\partial \bar{\phi}} \log W\right)^{2} \\
& =\frac{1}{8}\left(\frac{d \bar{\phi}}{d A}\right)^{2}=\frac{1}{8} \beta^{2},
\end{aligned}
$$

where $\beta=\frac{d \bar{\phi}}{d A}$ is the holographic beta function introduced in [10]. One can remove this factor by rescaling $h$

$h=\frac{8}{\beta} s=8 \sqrt{-\frac{2\left(\partial_{0} A\right)^{2}}{3 \partial_{0}^{2} A}} s$. 
The new field $s$ is a scalar with proper transformation properties under reparametrizations, however, it has a complicated potential $U\left(x_{0}\right)$, and is described by the action

$$
\begin{aligned}
S_{2}= & -\int d x_{0} d^{4} p \frac{1}{2} e^{4 A} \\
& \times\left(\left(\partial_{0} s\right)^{2}+\left(e^{-2 A} p^{2}+U\left(x_{0}\right)\right) s^{2}\right),
\end{aligned}
$$

where

$$
\begin{aligned}
U\left(x_{0}\right)= & 2\left(\frac{A^{\prime \prime}}{A^{\prime}}\right)^{2}-2 \frac{A^{\prime \prime \prime}}{A^{\prime}}-\frac{1}{4}\left(\frac{A^{\prime \prime \prime}}{A^{\prime \prime}}\right)^{2}+\frac{1}{2} \frac{A^{(i v)}}{A^{\prime \prime}} \\
& +2 \frac{A^{\prime \prime \prime} A^{\prime}}{A^{\prime \prime}}-4 A^{\prime \prime} .
\end{aligned}
$$

The appearance of the relative factor between $h$ and $s$ is indeed very natural, because the trace of the graviton $h$ is dual to the trace of the stress tensor and, as was discussed in [22], in a deformed conformal field theory we expect to have an operator relation [25]

$T_{i}^{i}(x)=\beta \mathscr{O}(x)$,

where $\mathscr{O}$ is the operator responsible for the deformation of the conformal field theory. In other words, the coupling of $h$ to the trace of the stress-energy tensor is $\int_{\varepsilon} h T=\int_{e} \frac{1}{\beta} s T \equiv \int_{\varepsilon} s \mathscr{O}$. Therefore, we see that if $h$ is dual to the trace of the stress tensor then the scalar $s$ is dual to the operator $\mathscr{O}$.

It is worth noting that we have no linear term in the final action. Linear terms do not appear because they can come only from total-derivative bulk terms, but we omit any such a term following the prescription of [23]. Moreover, according to [23], we do not need to add any boundary term to the actions (2.19) or (2.20), and these actions are appropriate for computing the 2-point function of the operator $\mathscr{O}$. The absence of linear terms leads to the vanishing of the one-point correlator of the operator $\mathscr{O}$ dual to the scalar $s$. This may look strange because one usually says that in a perturbed conformal field theory an operator dual to an active scalar has a nonvanishing one-point function. Nevertheless, we work in flat 4-dimensional space, and, therefore, we can always choose such a substraction scheme that a one-point function of any local operator vanishes. This seems to mean that the scalar $s$ is actually dual to the operator $\mathscr{O}-\langle\mathscr{O}\rangle$.
Deriving the action (2.19), we have not used the constraint (2.9) yet. One may wonder if this constraint imposes additional restrictions on admissible configurations of the gravity fields. In the Appendix we show that this constraint follows from the equations of motion for $h$ and $H$, and from the $h_{0 i}$-constraints (2.8), and, therefore, can be omitted.

Next we use the action (2.19) to compute 2-point functions in the two cases recently studied in [22]. Recall that the 2-point functions of active scalars obtained in [22] appear to be problematic. We will see that action (2.19) in both cases leads to reasonable 2-point functions.

We begin with the case considered in Section 3 of [22]. The supergravity solution discussed there was found in [11], and describes the renormalization group flow of $\mathscr{N}=4 \mathrm{SYM}$ theory to $\mathscr{N}=1 \mathrm{SYM}$ theory at long distances. We refer the reader to [11] for details.

To simplify the equations of motion of the scalar field and its solution, a new coordinate $u$ was introduced in [22] such that

$e^{2 A}=\frac{u}{1-u}, \quad \frac{d u}{d x_{0}}=\frac{2}{L}(1-u), \quad \frac{d A}{d x_{0}}=\frac{1}{L u}$.

In this coordinate the boundary of the 5-d space is at $u=1$, and there is a singularity at $u=0$. With these formulas we rewrite the action (2.19) as

$$
\begin{aligned}
S_{2} & =-\frac{3}{64 L} \int d u d^{4} p u^{2}\left(\left(\partial_{u} h\right)^{2}+\frac{p^{2} L^{2}}{4 u(1-u)} h^{2}\right) \\
& =-\frac{3}{64 L} \int d u d^{4} p \partial_{u}\left(u^{2} h \partial_{u} h\right),
\end{aligned}
$$

where in the second step the equation of motion for $h$

$\partial_{u}^{2} h+\frac{2}{u} \partial_{u} h-\frac{p^{2} L^{2}}{4 u(1-u)} h=0$

has been used. To compute the 2-point function we follow the standard AdS/CFT prescription [2,3]. Imposing Dirichlet boundary conditions at $u=1-$ $\varepsilon^{2}$, we obtain the solution to (2.23), regular at $u=0$

$h(u, p)=\frac{F\left(a_{-}, a_{+} ; 2 ; u\right)}{F\left(a_{-}, a_{+} ; 2 ; 1-\varepsilon^{2}\right)} h(p)$, 
where

$a_{ \pm}=\frac{1}{2}\left(1 \pm \sqrt{1-p^{2} L^{2}}\right)$

and $F(a, b ; c, u)$ is the hypergeometric function ${ }_{2} F_{1}$. The 2-point function of $h$ in momentum space is given by the familiar formula

$$
\begin{aligned}
& \langle O(p) O(-p)\rangle \\
& \quad=\left.\lim _{\varepsilon \rightarrow 0} \frac{3}{32 L} \frac{\partial_{u} F\left(a_{-}, a_{+} ; 2 ; u\right)}{F\left(a_{-}, a_{+} ; 2 ; u\right)}\right|_{u=1-\varepsilon^{2}}
\end{aligned}
$$

To find the 2-point function we need

$$
\begin{aligned}
& \frac{d}{d u}(u F(a, b ; 2 ; u))=F(a, b ; 1 ; u), \\
& F\left(a_{-}, a_{+} ; 2 ; 1\right)=\frac{1}{\Gamma\left(2-a_{-}\right) \Gamma\left(2-a_{+}\right)},
\end{aligned}
$$

and the expansion

$$
\begin{aligned}
F\left(a_{-}, a_{+} ; 1 ; u\right)= & \frac{1}{\Gamma\left(a_{-}\right) \Gamma\left(a_{+}\right)} \\
& \times\left(2 \Psi(1)-\Psi\left(a_{-}\right)-\Psi\left(a_{+}\right)\right. \\
& -2 \log \varepsilon+o(\varepsilon)) .
\end{aligned}
$$

Then, omitting all terms polynomial in $p$, we find in the limit $\varepsilon \rightarrow 0$

$$
\begin{aligned}
& \langle O(p) O(-p)\rangle \\
& =\frac{3}{32 L} \frac{p^{2} L^{2}}{4}\left(\Psi\left(\frac{1}{2}\left(1-\sqrt{1-p^{2} L^{2}}\right)\right)\right. \\
& \left.\quad+\Psi\left(\frac{1}{2}\left(1+\sqrt{1-p^{2} L^{2}}\right)\right)\right) .
\end{aligned}
$$

The correlator has a discrete spectrum of poles at $-p^{2}=4 n(n+1) / L^{2}, \quad n=0,1, \ldots$ A similar discrete spectrum was found in $[11,22]$ by studying 2-point correlators of inert scalars. Thus contrary to the result in [22], we obtain for the active scalar a 2-point correlator with the expected behaviour.

Next we consider the second case, cf. Section 4 of [22], where the flow obtained in $[18,19]$ was used to analyze the 2-point function of an active scalar. This flow changes the vacuum of $\mathscr{N}=4$ SYM which is now on the Coulomb branch.

A new coordinate $v$ was introduced [22] such that $e^{2 A}=\frac{l^{2}}{L^{2}} \frac{v^{2 / 3}}{1-v}, \quad \frac{d v}{d x_{0}}=\frac{2}{L} v^{2 / 3}(1-v)$,

$\frac{d A}{d x_{0}}=\frac{v+2}{3 L v^{1 / 3}}$,

$l$ is an additional length scale. As in the first example, the boundary of the 5-d space is at $v=1$, and there is a singularity at $v=0$.

First we check that our interpretation of the field $s$ as the dual to the operator $O_{2}$ in the $\mathrm{YM}$ is consistent. For the kink solution (2.27) there exists a limiting procedure [18] that allows one to remove the flow and to restore the AdS solution. This is a limiting case when $\frac{l^{2}}{L^{2}} \equiv \xi^{2}$ becomes small while $\frac{z}{L}=x$ is kept fixed. The variable $z$ is introduced via

$$
\begin{aligned}
v & =\operatorname{sech}^{2}\left(\frac{z l}{L^{2}}\right) \\
& =\frac{1}{\operatorname{ch}^{2}(\xi x)}=1-\xi^{2} x^{2}+\frac{2}{3} \xi^{4} x^{4}+\ldots
\end{aligned}
$$

Clearly, in this limit

$2 A=\log \left(\xi^{2} \frac{v^{2 / 3}}{1-v}\right) \approx-\log \left(x^{2}\right)$,

and from $\frac{d v}{d x_{0}}=\frac{2}{L} v^{2 / 3}(1-v)$ it follows that

$d x_{0}=-\frac{L}{x} d x$,

i.e. one recovers the standard AdS metric.

By using $A^{\prime}=d A / d x_{0}$ from (2.27) together with (2.28) it is easy to find the leading terms of the derivatives

$$
\begin{aligned}
& A^{\prime} \sim \frac{1}{L}+\frac{1}{9 L} \xi^{4} x^{4}, \quad A^{\prime \prime} \sim-\frac{4}{9 L^{2}} \xi^{4} x^{4}, \\
& A^{\prime \prime \prime} \sim \frac{4^{2}}{9 L^{3}} \xi^{4} x^{4}, \quad A^{(I V)} \sim-\frac{4^{3}}{9 L^{4}} \xi^{4} x^{4} .
\end{aligned}
$$

Since $\beta \sim \xi^{2}$, it vanishes in this limit. 
Upon substituting (2.29) into (2.21) one gets $U\left(x_{0}\right)=-\frac{4}{L^{2}}+\mathscr{O}\left(\xi^{4}\right)$. Thus, in the limit $\xi \rightarrow 0$ the action (2.20) becomes

$$
\begin{aligned}
S_{2}= & -\frac{L^{3}}{2} \int d z d^{4} p \sqrt{-g_{a}} \\
& \times\left(z^{2}\left(\partial_{z} s\right)^{2}+z^{2} p^{2} s^{2}-4 s^{2}\right),
\end{aligned}
$$

where $g_{a}$ is the determinant of the standard $A d S$ metric. Eq. (2.30) is the familiar action for the scalar field on the AdS space with mass $m^{2}=-4$ that is dual to the YM operator $\mathrm{O}_{2}$ of conformal weight $\Delta=2$. It is worth noting that from the point of view of the 2-point correlator of the operator dual to the scalar $s$, the limit $\xi \rightarrow 0$ is equivalent to taking $p^{2}$ to infinity, i.e. to the UV limit. Therefore, this consideration shows that the 2-point correlator does not vanish, and behaves itself in the UV as expected from an operator of the UV conformal dimension $\Delta=2$.

With the help of (2.27) we rewrite (2.20) as

$$
\begin{aligned}
S_{2}= & -\frac{l^{4}}{L^{5}} \int d v d^{4} p \frac{v^{2}}{(1-v)} \\
& \times\left(\left(\partial_{v} s\right)^{2}+\frac{p^{2} L^{4}}{4 l^{2} v^{2}(1-v)} s^{2}\right. \\
& \left.-\frac{3\left(4-2 v+v^{2}\right)}{(v+2)^{2} v(1-v)^{2}} s^{2}\right) .
\end{aligned}
$$

This action leads to the following equation of motion for $s$

$$
\begin{gathered}
\partial_{v}^{2} s+\frac{2-v}{v(1-v)} \partial_{v} s-\frac{p^{2} L^{4}}{4 l^{2} v^{2}(1-v)} s \\
+\frac{3\left(4-2 v+v^{2}\right)}{(v+2)^{2} v(1-v)^{2}} s=0 .
\end{gathered}
$$

The on-shell value of the action is

$$
S_{2}=-\frac{l^{4}}{L^{5}} \int d^{4} p\left(\frac{v^{2}}{1-v} s \partial_{v} s\right)_{v=1-\varepsilon^{2}} .
$$

Eq. (2.32) has four regular singular points, $(-2,0,1, \infty)$ and a closed form solution does not exist. But we can nevertheless analyze the behaviour of a solution in the neighbourhood of the physically relevant points at $v=1$ and at $v=0$. At $v=1$, the power series Ansatz $s(v)=(1-v)^{\rho}\left[1+a_{1}(1-v)\right.$
$\left.+\mathscr{O}\left((1-v)^{2}\right)\right]$ leads to the indicial equation $\rho^{2}-$ $2 \rho+1=0$ with two degenerate solutions $\rho=1$. Thus, the general solution is of the form

$$
s(v)=(1-v)\left(c_{1} f_{1}(v)+c_{2} f_{2}(v) \log (1-v)\right) \text {, }
$$

where $f_{1}$ and $f_{2}$ are power series in $(1-v)$ with leading term 1 .

A similar analysis at $v=0$ leads to an indicial equation with the two solutions

$\rho_{ \pm}=-\frac{1}{2} \pm \frac{1}{2} \sqrt{1+\frac{p^{2} L^{4}}{l^{2}}}$.

For generic parameters the two independent solutions are pure power series. Of these, the one for $\rho_{-}$, is forbidden by regularity. Thus, the solution we are interested in has the general form

$h(v)=c v^{\rho_{+}}\left(1+a_{1} v+a_{2} v^{2}+\ldots\right)$.

It is regular at $v=0$ for space-like momenta $\left(p^{2}>\right.$ 0 ). Note however that although the solution is not regular at the singularity $v=0$ if the momentum obeys $0 \leq-p^{2}<l^{2} / L^{4}$, the $v$-integration, cf. (2.33), gives a vanishing contribution at $v=0$ due to the factor $v^{2}$ in the numerator.

To find the 2-point function we need to analytically continue this solution to the neighbourhood of $v=1$. By comparing with the known solution of (2.32) in the UV limit $p^{2} \rightarrow \infty(\xi \rightarrow 0)$ we conclude that both constants $c_{1}$ and $c_{2}$ in (2.34) are nonvanishing. It is not difficult to compute the 2-point function in terms of these constants. By using the conventional rules of the AdS/CFT correspondence, we get

$$
\begin{aligned}
& \langle O(p) O(-p)\rangle \\
& \quad=\left.\lim _{\varepsilon \rightarrow 0} \frac{2 l^{4}}{L^{5}} \frac{v^{2}}{1-v} \partial_{v} s_{\varepsilon}(v)\right|_{v=1-\varepsilon^{2}},
\end{aligned}
$$

where

$s_{\varepsilon}(v)=\frac{(1-v)\left(c_{1} f_{1}(v)+c_{2} f_{2}(v) \log (1-v)\right)}{\varepsilon^{2}\left(c_{1} f_{1}\left(1-\varepsilon^{2}\right)+c_{2} f_{2}\left(1-\varepsilon^{2}\right) \log \left(\varepsilon^{2}\right)\right)}$

is the solution of the equation of motion normalized to be 1 at $v=1-\varepsilon^{2}$. The leading term in $\varepsilon$, non-analytic in $p^{2}$, is

$$
\langle O(p) O(-p)\rangle=\frac{1}{\varepsilon^{4} \log ^{2} \varepsilon} \cdot \frac{l^{4} c_{1}}{2 L^{5} c_{2}} .
$$


The factor $\frac{1}{\varepsilon^{4} \log ^{2} \varepsilon}$ is the one that one expects in the 2-point correlator of a scalar field with the UV conformal weight 2 (see, e.g. [26] or the Appendix of [27]). Although we do not know the constants $c_{1}$ and $c_{2}$ explicitly, their $p^{2}$-dependence will be through $\rho_{+}$. We thus conclude, as in [22] for the case of the inert scalar, that the correlator has a continuous spectrum with a mass gap, $m^{2} \geq l^{2} / L^{4}$, which vanishes in the limit $\xi \rightarrow 0$.

\section{Conclusion}

In this paper we studied graviton-scalar fluctuations in $d=5$ flow geometries which are dual to boundary field theories with RG flow. We showed that the analysis of the coupled gravity-scalar sector drastically simplifies by a choice of the almost radial gauge, where one of the active scalars vanishes, but the trace of the graviton $h$ remains dynamical. We considered in detail the simplest case of one active scalar, decoupled the graviton trace from the transverse traceless components, and obtained a very simple quadratic action for it. The Lagrangian of the graviton trace differs from the one of a minimallycoupled massless scalar field (e.g. dilaton) only by a factor which coincides with the square of the holographic beta function of the operator responsible for the deformation of the conformal field theory. This is a very natural result due to the operator relation $T_{i}^{i}=\sum_{I} \beta^{I} \mathscr{O}^{I}$ in a deformed CFT. We expect that a similar relation between the action for $h$ and the dilaton action also holds for the general case of many active scalars, where the factor would be given by the square of a "weighted" holographic beta function.

We fixed the form of the quadratic action by means of the Hamiltonian prescription [23] and used the action to compute 2-point functions of the graviton trace in two cases of flow geometries. In both cases we obtained physically reasonable functions which have the same momentum dependence as those of inert scalars. Thus, we successfully resolved the problem recently raised in [22].

It is worth noting that the geometries we considered have a curvature singularity in the interior and one would expect large string corrections to the 2-point functions, which could drastically change the behaviour of the correlators. Nevertherless, this seems not to happen because the curvature is small near the boundary, and the contribution of the vicinity of the singularity to the on-shell gravity action vanishes.

\section{Acknowledgements}

We would like to thank A. Tseytlin for useful comments. The work of G.A. was supported by the Alexander von Humboldt Foundation and in part by the RFBI grant N99-01-00166, and the work of S.F. was supported by the US Department of Energy under grant No. DE-FG02-96ER40967 and in part by RFBI grant N99-01-00190. S.T. is supported by GIF - the German-Israeli Foundation for Scientific Research by the TMR programme ERBRMX-CT960045 .

\section{Appendix A. The second constraint}

Here we analyze the constraint (2.9) and show that it follows from (2.8) and the equations of motion. Since the equation of motion for $\bar{\phi}$ is

$$
\frac{\partial V}{\partial \bar{\phi}}=\partial_{0}^{2} \bar{\phi}+4 \partial_{0} \bar{\phi} \partial_{0} A \text {, }
$$

Eq. (2.9) can be written as

$$
C=\partial_{0} h_{00} \partial_{0} \bar{\phi}+2 \frac{\partial V}{\partial \bar{\phi}} h_{00}-\partial_{0} t \partial_{0} \bar{\phi} \text {. }
$$

By using the equation of motion for $h_{00}$ together with (2.8) we find

$p^{2} \partial_{0} H=-\partial_{0} h-\frac{V}{3} \frac{\partial_{0} h}{\left(\partial_{0} A\right)^{2}}+\frac{1}{4} \frac{e^{-2 A} p^{2} h}{\partial_{0} A}$

and, therefore,

$\partial_{0} t=-\frac{V}{3} \frac{\partial_{0} h}{\left(\partial_{0} A\right)^{2}}+\frac{1}{4} \frac{e^{-2 A} p^{2} h}{\partial_{0} A}$.

Taking into account that

$\partial_{0} h_{00}=\frac{1}{4} \frac{\partial_{0}^{2} h}{\partial_{0} A}-\frac{1}{4} \frac{\partial_{0}^{2} A}{\left(\partial_{0} A\right)^{2}} \partial_{0} h$, 
one finds

$$
\begin{aligned}
C= & \left(\frac{1}{4} \frac{\partial_{0}^{2} h}{\partial_{0} A}-\frac{1}{4} \frac{\partial_{0}^{2} A}{\left(\partial_{0} A\right)^{2}} \partial_{0} h-\frac{1}{4} \frac{e^{-2 A} p^{2} h}{\partial_{0} A}\right. \\
& \left.+\frac{V}{3} \frac{\partial_{0} h}{\left(\partial_{0} A\right)^{2}}\right) \partial_{0} \bar{\phi}+\frac{1}{2} \frac{\partial V}{\partial \bar{\phi}} \frac{\partial_{0} h}{\partial_{0} A}
\end{aligned}
$$

From (2.19) one finds the equation of motion for $h$ :

$$
\begin{gathered}
\frac{\partial_{0}^{2} h}{\partial_{0} A}-2 \frac{\partial_{0}^{2} A}{\left(\partial_{0} A\right)^{2}} \partial_{0} h-\frac{e^{-2 A} p^{2} h}{\partial_{0} A} \\
=-4 \partial_{0} h-\frac{\partial_{0}^{3} A}{\partial_{0} A \partial_{0}^{2} A} \partial_{0} h .
\end{gathered}
$$

Therefore, by virtue of this equation one obtains

$$
\begin{gathered}
C=\left(\frac{1}{4} \frac{\partial_{0}^{2} A}{\left(\partial_{0} A\right)^{2}} \partial_{0} h-\partial_{0} h-\frac{\partial_{0}^{3} A}{4 \partial_{0} A \partial_{0}^{2} A} \partial_{0} h\right. \\
\left.+\frac{V}{3} \frac{\partial_{0} h}{\left(\partial_{0} A\right)^{2}}\right) \partial_{0} \bar{\phi}+\frac{1}{2} \frac{\partial V}{\partial \bar{\phi}} \frac{\partial_{0} h}{\partial_{0} A} .
\end{gathered}
$$

Recalling (2.12), one then finds

$$
\begin{aligned}
C= & \frac{1}{4}\left(-8-\frac{\partial_{0}^{3} A}{\partial_{0} A \partial_{0}^{2} A}\right) \partial_{0} \bar{\phi} \partial_{0} h \\
& +\frac{1}{2} \frac{\partial V}{\partial \bar{\phi}} \frac{\partial_{0} h}{\partial_{0} A} .
\end{aligned}
$$

Differentiating (2.12), one gets

$$
\partial_{0}^{3} A+8 \partial_{0} A \partial_{0}^{2} A+\frac{4}{3} \frac{\partial V}{\partial \bar{\phi}} \partial_{0} \bar{\phi}=0
$$

and, as a consequence,

$$
\begin{aligned}
C & =\frac{1}{3} \frac{\partial V}{\partial \bar{\phi}} \frac{\left(\partial_{0} \bar{\phi}\right)^{2} \partial_{0} h}{\partial_{0} A \partial_{0}^{2} A}+\frac{1}{2} \frac{\partial V}{\partial \bar{\phi}} \frac{\partial_{0} h}{\partial_{0} A} \\
& =\frac{1}{2} \frac{\partial V}{\partial \bar{\phi}} \frac{\partial_{0} h}{\partial_{0} A \partial_{0}^{2} A}\left(\frac{2}{3}\left(\partial_{0} \bar{\phi}\right)^{2}+\partial_{0}^{2} A\right) .
\end{aligned}
$$

Finally, we have the relation

$$
\partial_{0}^{2} A=-\frac{g}{3} \frac{\partial W}{\partial \phi} \partial_{0} \bar{\phi}=-\frac{2}{3}\left(\partial_{0} \bar{\phi}\right)^{2} \text {. }
$$

Upon substituting this relation into the previous formula we find $C=0$. Thus, constraint (2.9) is compatible with the dynamics.

\section{References}

[1] J. Maldacena, Adv. Theor. Math. Phys. 2 (1998) 231.

[2] G.G. Gubser, I.R. Klebanov, A.M. Polyakov, Phys. Lett. B 428 (1998) 105, hep-th/9802109.

[3] E. Witten, Adv. Theor. Math. Phys. 2 (1998) 253, hepth/9802150.

[4] L. Girardello, M. Petrini, M. Porrati, A. Zaffaroni, JHEP 12 (1998) 022, hep-th/9809047.

[5] J. Distler, F. Zamora, Adv. Theor. Math. Phys. 2 (1999) 1405, hep-th/9810206.

[6] A. Khavaev, K. Pilch, N.P. Warner, New vacua of gauged $\mathscr{N}=8$ supergravity in five dimensions, hep-th/9812035.

[7] A. Karch, D. Lust, A. Miemiec, Phys. Lett. B 454 (1999) 265, hep-th/9901041.

[8] D.Z. Freedman, S.S. Gubser, K. Pilch, N.P. Warner, Renormalization group flows from holography-supersymmetry and a c-theorem, hep-th/9904017.

[9] L. Girardello, M. Petrini, M. Porrati, A. Zaffaroni, JHEP 05 (1999) 026, hep-th/9903026.

[10] D. Anselmi, L. Girardello, M. Porrati, A. Zaffaroni, A note on the holographic beta and $c$ functions, hep-th/0002066.

[11] L. Girardello, M. Petrini, M. Porrati, A. Zaffaroni, The supergravity dual of $\mathscr{N}=1$ Super Yang-Mills Theory, hepth/9909047.

[12] S.S. Gubser, Non-conformal examples of AdS/CFT, hepth/9910117.

[13] J. de Boer, E. Verlinde, H. Verlinde, On the Holographic Renormalization Group, hep-th/9912012.

[14] I. Klebanov, N. Nekrasov, Gravity duals of fractional branes and logarithmic RG flow, hep-th/9911096.

[15] I. Klebanov, A. Tseytlin, Gravity Duals of Supersymmetric $S U(N) \times S U(N+M)$ Gauge Theories, hep-th/0002159.

[16] P. Kraus, F. Larsen, S.P. Trivedi, JHEP 03 (1999) 003, hep-th/9811120.

[17] I.R. Klebanov, E. Witten, Nucl. Phys. B 556 (1999) 89, hep-th/9905104.

[18] D.Z. Freedman, S.S. Gubser, K. Pilch, N.P. Warner, Continuous distributions of D3-branes and gauged supergravity, hep-th/9906194.

[19] A. Brandhuber, K. Sfetsos, Wilson loops from multicentre and rotating branes, mass gaps and phase structure in gauge theories, hep-th/9906201.

[20] M. Cvetic, S. S Gubser, H. Lu, C. Pope, Symmetric potentials of gauged supergravities in diverse dimensions and Coulomb branch of gauge theories, hep-th/9909121.

[21] S.S. Gubser, Curvature singularities: the good, the bad, and the naked, hep-th/0002160. 
[22] O. DeWolfe, D.Z. Freedman, Notes on fluctuations and correlation functions in holographic renormalization group flows, hep-th $/ 0002226$.

[23] G. Arutyunov, S. Frolov, Nucl. Phys. B 544 (1999) 576, hep-th/9806216.

[24] G. Arutyunov, S. Theisen, S. Frolov, work in progress.
[25] H. Osborn, Nucl. Phys. B 363 (1991) 486.

[26] P. Minces, V.O. Rivelles, Scalar field theory in the AdS /CFT correspondence revisited, hep-th/9907079.

[27] G. Arutyunov, S. Frolov, Four-point Functions of Lowest Weight CPOs in $\mathscr{N}=4 \mathrm{SYM}_{4}$ in Supergravity approximation, hep-th/0002170. 